\title{
Design of a Smart Military Fatigue
}

\author{
Ogboru OR ${ }^{1 *}$, Adebayo $\mathrm{OT}^{2}$ and Adebayo FO $^{1}$ \\ ${ }^{1}$ Department of Computer Science, Federal University of Technology, Akure \\ ${ }^{2}$ Department of Information Technology, Federal University of Technology, Akure
}

*Corresponding author: Ogboru OR, Department of Computer Science, Federal University of Technology, Akure

\begin{abstract}
Locating troops and early reporting of injuries sustained in the battlefield and rapid identification of personnel (ally or enemy) almost instantaneously has become a crucial part of modern warfare. Accurate identification, quick medical support and location tracking of own troops not only saves lives of personnel but also helps troops in on-time effective engagement of enemy forces while also providing reinforcement, troops extraction in cases where they are overwhelmed by enemy forces. The Smart Military Fatigue is equipped with sensors such. Each personnel are equipped with a tag which will contain basic identification information such as name, unique ID number, Rank, Designation, Callsign etc., these tags will replace conventional identification cards and must be carried at all times. Whenever an unknown target is in sight, the reader is targeted onto such target and then the tag sends the information stored onto its memory to the reader thereby displaying useful identification details as mentioned above. The details gotten from the tag are then compared to a centralized database to effectively and accurately identify an unknown threat.
\end{abstract}

Keywords: RFID; GPS; Proteus

\section{Introduction}

Personnel identification has solely relied on human intelligence, command signals through radio (which can be intercepted by enemy forces) but these often fail as enemy forces can dress in own uniform thereby leading to ambush which are usually deadly and causes heavy casualties, therefore, a promising technology that can mitigate such constraints is Radio frequency identification (RFID) for personnel identification and Global positioning system (GPS) for real-time personnel tracking. Ultra-high (UHF) RFID tags are placed on military fatigue which are then scanned by weaponmounted RFID readers which may not require direct line of sight to the tags for instant identification, the GPS system constantly logs troops location. RFID stands for Radio-Frequency Identification. The acronym refers to small electronic devices that consist of a small hip and an antenna. The chip typically is capable of carrying 2,000 bytes of data or less. RFID is a general term that is used to describe a system that transmits the identity (in the form of a unique serial number) of an object wirelessly, using radio waves. This is sometimes referred to as contact-less technology and a typical RFID system is made up of three components: tags, readers and the host computer system. An RFID tag is a tiny radio device that is also referred to as a transponder, smart tag, smart label or radio barcode. The tag comprises of a simple silicon microchip attached to a small flat aerial and mounted on a substrate. The whole device can then be encapsulated in different materials (such as plastic) dependent upon its intended usage. The finished tag can be attached to an object, typically an item, box or pallet and read remotely to ascertain its identity, position or state. This project is designed to help the Defense industry mitigate the loss of personnel which may occur from ambushes by enemy forces or engagement by two friendly forces who may engage themselves due to difficulty in quick identification (referred to as Blue-onBlue). A smart tag containing the basic details of each personnel such as army id number, unit designation, callsign, or a unique ID etc., are pre-programmed onto the tag and is worn on the personnel. When the RFID reader which may be mounted on the scope of a rifle, binoculars or tactical glasses scans the personnel and then reveals the details aforementioned above thereby acting as a decision support system; the system receives the details from the tag and checks across a database, if not found, it displays enemy contact otherwise it displays friendly on an LCD. The GPS module is also worn on-body which constantly logs the location of troops real-time from central command. 


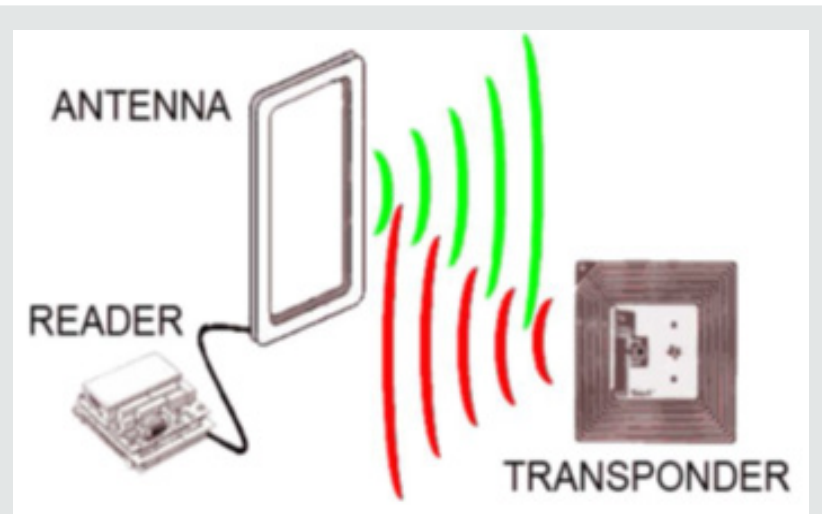

Figure 1: RFID reader and tag.

System implementation and simulation of result: Figure 2

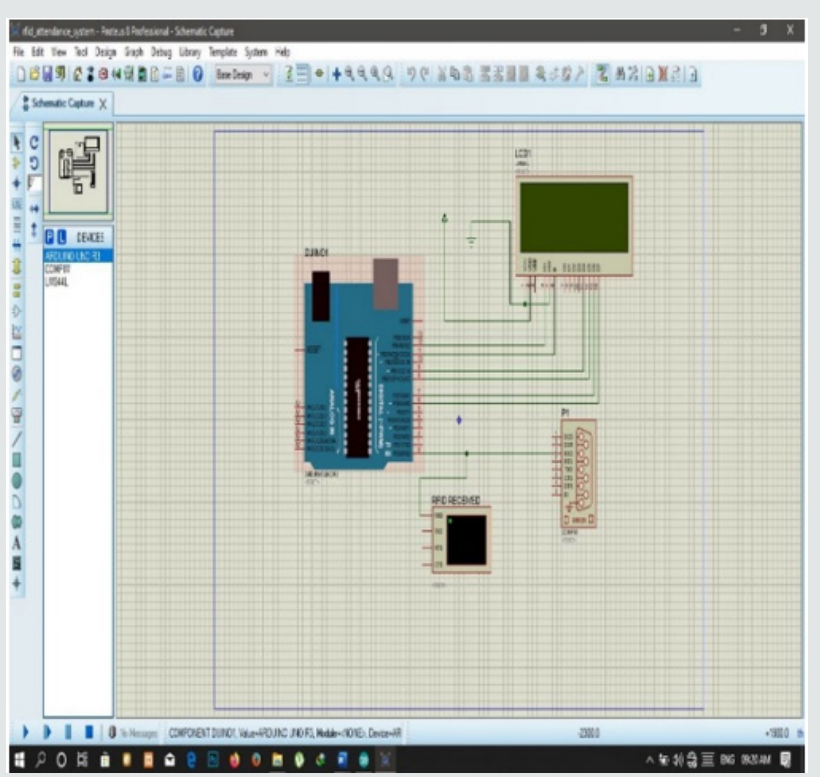

Figure 2: RFID simulation circuit.

\section{Related Work}

The paper [1] presented location of troops using Zigbee tags, routers and gateways for effectively reporting location of troops who strayed away from their units after a contact had been made with an enemy. Sensors were integrated into the combat fatigue of personnel which actively monitors the current geolocation of troops and reported same to an overhead command which then sends an extraction team to troops. Paper [2] presented an RFID based intelligent book finder using an Ultra High Frequency sensor. In this paper, tags were fitted into books in a library which upon being scanned by an RFID reader are able to check when a book was borrowed, who borrowed it, due date to be returned (Figure 1). Paper [3] presented a Smart ID card system using RFID technology where the system will allow the personnel in charge at a college or university to easily track student money transactions and also allow to make attendance in the easiest manner. The administrator will be able to make reports easily by one simple click [4].

\section{Proposed System}

This paper proposes the use of RFID technology for the identification of troops. RFID technology is a form of technology that is used for object identification such as books in an inventory, it can be used for tracking inventories in a warehouse amongst numerous other uses. RFID technology has advantages over other technology such as Barcode in that it does not require a direct line-of-sight [5]. This paper proposes that UHF tags are placed into the military fatigue of troops and must be worn at all times. When a reader is targeted on it, the tags sends details pre-programmed into the tags to an LCD which displays information used for identification.

\section{Software Description}

To implement the Smart Military Fatigue, a platform has been selected which includes the Software: Embedded C, PIC C Compiler, Proteus design suite.

\section{Proteus Design Suits}

The Proteus Design Suite is an Electronic Design Automation (EDA) tool including schematic capture, simulation and PCB Layout modules. It can be purchased in many configurations, depending on the size of designs being produced and the requirements for microcontroller simulation. Schematic capture in the Proteus Design Suite is used for both the simulation of designs and as the design phase of a PCB layout project. It is therefore a core component and is included with all product configurations. The micro-controller simulation in Proteus works by applying either a hex file or a debug file to the microcontroller part on the schematic. Proteus is composed of two applications called ARES and ISIS. ISIS is mainly focused on the design of a real circuit board and how it works with the different elements that a PCB has. For example, it allows you to simulate microcontrollers and its different connections in real time [6]. On the other hand, ARES is able to route and place the different objects and is used to generate printed circuit boards. Besides that, one of the good things about this program is that it is very versatile and is able to effectively integrate all the parts of the project. Proteus incorporates a common database which allows the user to automatically update the data of the different modules which are part of the main project. In addition, the 3D viewer has been improved and lets you see changes and modifications in [7].

\section{A Flowchart}

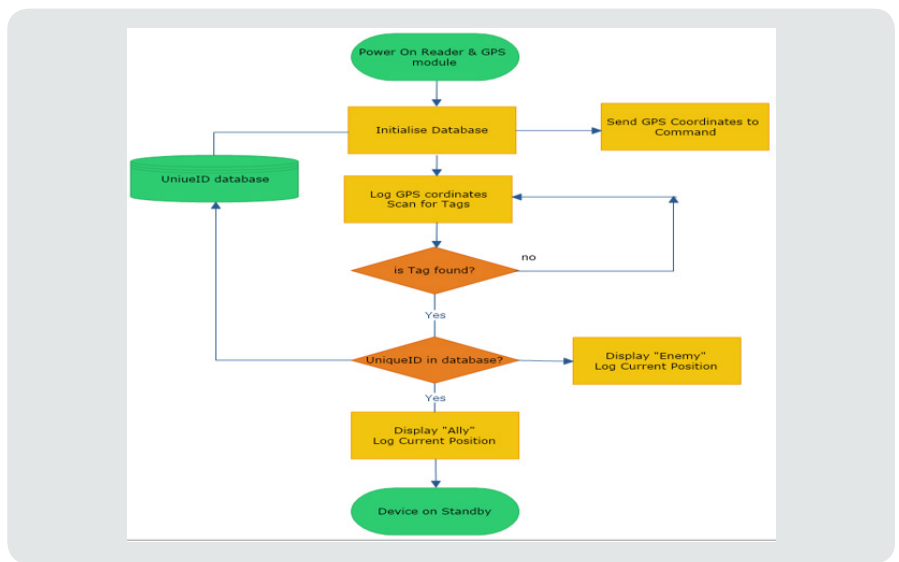




\section{System Design}

Arduino system can be used to implement this scheme because it is relatively small and can easily be embedded into the clothing, it is cheap to implement on a large scale, its standby time is relatively high and can be powered from tiny cell battery (Figure 3-5). The RFID system is mounted on the scope of a rifle, binocular, smart glass or any other instrument that is capable of seeing objects in a distance. The tags that are pre-programmed with details such as name, unique id, designation, callsign etc., are embedded into the fabric of the fatigue are worn by the soldiers. When a reader is focused on the fatigue, the tags will power itself from the electromagnetic force of the reader causing the tag to send preprogrammed information already stored in it. The GPS module is also embedded into the fatigue which actively logs and sends troops current and last known location to s central command [8].

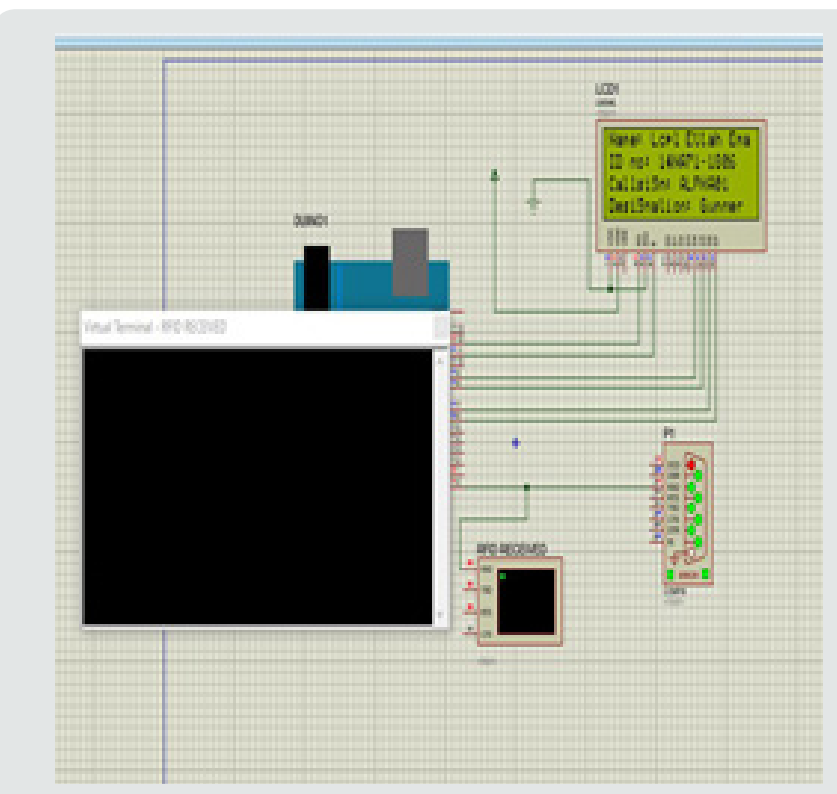

Figure 3: RFID simulation showing a known contact.

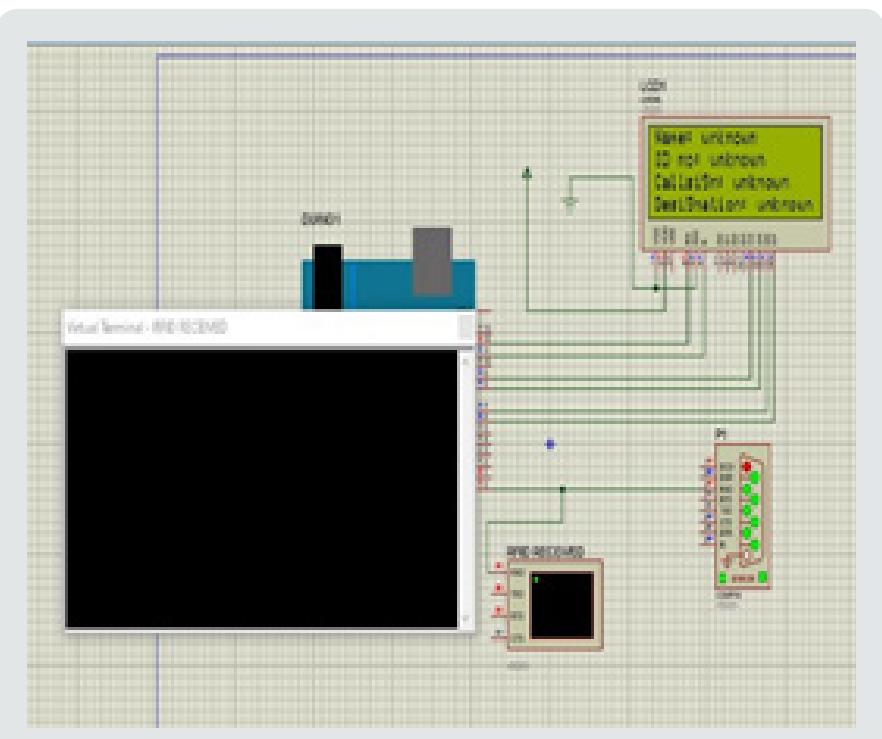

Figure 4: RFID system showing unknown target.

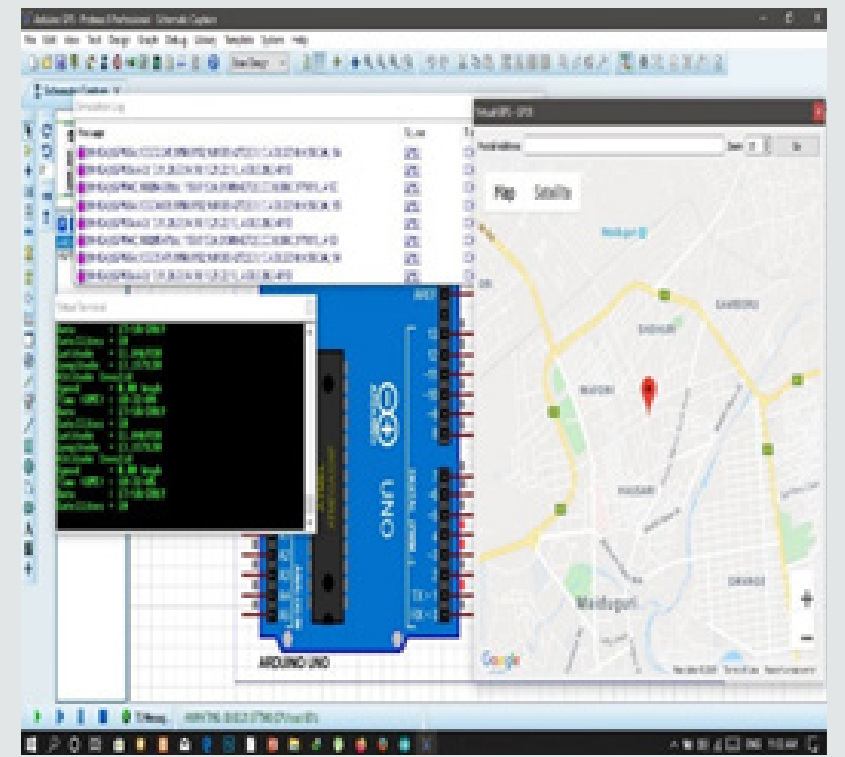

Figure 5: GPS module showing live feed.

\section{Conclusion}

The Smart Military Fatigue is intended for use by the military. The smart military fatigue will enable troops easily identify potential threats whom may dress in own uniform to lay ambush or surprise attacks by quickly and effectively displaying vital information such as name, picture, unit attached to, call sign etc., which will enable commanders make right decisions at critical moments.it will also enable command track and locate troops whom might be in distress. The RFID system is better than other types of object identification because it does not require a direct line of sight to its target thereby making it a viable solution.

\section{References}

1. Kang HJ, Lee D, Shin JG, Park BJ (2011) Location Tracking Of Moving Crew Members For Effective Damage Control In An Emergency 61(1): 57-61.

2. Nithya M, Solaiyammal K (2017) RFID Based Intelligent Book Finder Using Ultra High Frequency Sensor. International Journal of Advanced Research in Electrical Electronics and Instrumentation Engineering $5(5)$.

3. Leandre N, Addepalli S, Vivek V (2018) Smart ID card system using RFID technology. International Journal for Research in Applied Science \& Engineering Technology 6(5).

4. Roberts CM (2006) Radio Frequency Identification. Computers \& Security Elsevier 25(1): 18-26.

5. Jerry L, Barbara C (2001) Shrouds of Time: The History of RFID. AIM Publication.

6. Ayoub Khan M, Manoj S and Prahbu RB (2019) A Survey of RFID Tags. International Journal of Recent Trends in Engineering 1(4).

7. Wing WN, Yi Song Qiao, Lin L, Hai Lan Ding, Patrick PK, et al. (2011) Intelligent book positioning for library using RFID and book spine matching.

8. Narayanan A, Sanjay Singh, Somasekharan M (2010) Implementing RFID in Library: Methodologies Advantages and Disadvantages. pp. 271-281. 
(C) (P) This work is licensed under Creative

To Submit Your Article Click Here: Submit Article

DOI: $10.32474 /$ CTCSA.2019.01.000124

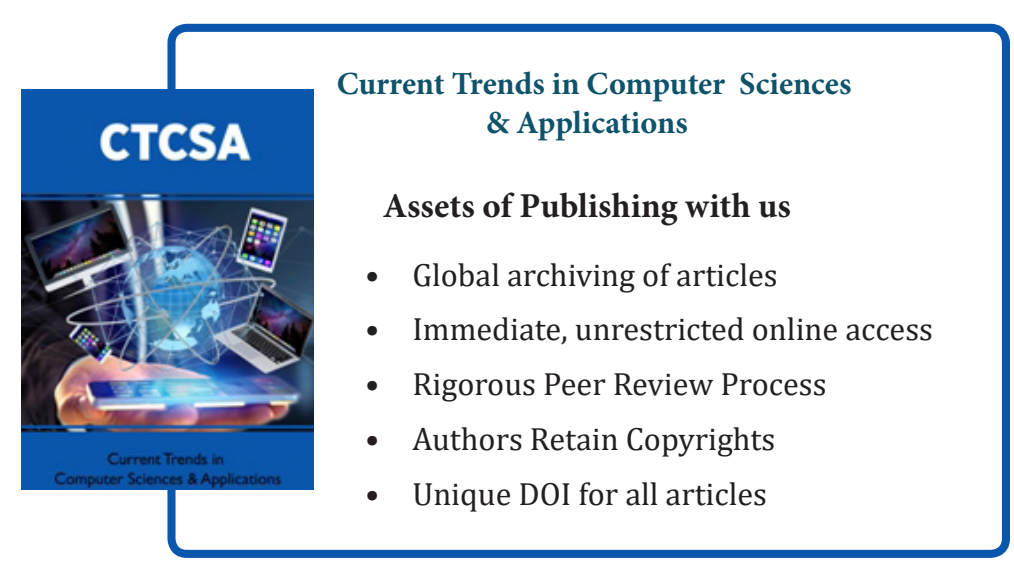

